\title{
A surprising relation for the effective coupling constants of $N=2$ super Yang-Mills theories
}

\author{
Marco Matone \\ Dipartimento di Fisica e Astronomia "G. Galilei", Istituto Nazionale di Fisica Nucleare, \\ Università di Padova, Via Marzolo, 8-35131 Padova, Italy \\ E-mail: matone@pd.infn.it
}

ABSTRACT: We show that the effective coupling constants $\tau$ of supersymmetric gauge theories described by hyperelliptic curves do not distinguish between the lattices of the two kinds of heterotic string. In particular, the following relation

$$
\Theta_{D_{16}^{+}}(\tau)=\Theta_{E_{8}}^{2}(\tau)
$$

holds. This is reminiscent of the relation, by $T$-duality, of the two heterotic strings. We suggest that such a relation extends to all curves describing effective supersymmetric gauge theories.

KeYwords: Supersymmetry and Duality, Nonperturbative Effects, Supersymmetric Effective Theories, String Duality

ARXIV EPRINT: 1504.00349 
Let us consider the Seiberg and Witten effective coupling constant $[1,2]$

$$
\tau_{i j}=\frac{\theta_{i j}}{\pi}+\left(\frac{8 \pi i}{g^{2}}\right)_{i j} .
$$

Positivity of $g^{2}$, which is crucial to guarantee unitarity of the theory, is a consequence of the Riemann bilinear relations, that imply

$$
\operatorname{Im} \tau_{i j}>0 \text {. }
$$

We now show that $\tau_{i j}$ satisfies another important relation.

The Seiberg-Witten theory, that in the simplest case has been proved in [3], is described by hyperelliptic curves $[4,5]$

$$
y^{2}=\prod_{k=1}^{2 g+2}\left(x-a_{i}\right) .
$$

The standard example is the case of gauge group $\mathrm{SU}(n)$ whose hyperelliptic curves have genus $g=n-1$.

A basic result for hyperelliptic curves follows by the polynomial identity

$$
\begin{aligned}
& \left(\sum_{\left\{T \amalg^{c}\right\}} \prod_{i<j ; i, j \in T}\left(a_{i}-a_{j}\right)^{2} \prod_{i<j ; i, j \in T^{c}}\left(a_{i}-a_{j}\right)^{2}\right)^{2} \\
& \quad=2^{g} \sum_{\left\{T \amalg^{c}\right\}} \prod_{i<j ; i, j \in T}\left(a_{i}-a_{j}\right)^{4} \prod_{i<j ; i, j \in T^{c}}\left(a_{i}-a_{j}\right)^{4},
\end{aligned}
$$

where the sum is over the $\frac{1}{2}\left(\begin{array}{c}2 g+2 \\ g+1\end{array}\right)$ partitions $T \coprod^{c}$ of $\{1, \ldots 2 g+2\}$ for which both $T$ and $T^{c}$ have $g+1$ elements. Such an identity, that holds for $g \geq 1$, and that can be proved by induction by letting $a_{2 g+1}=a_{2 g+2}$, has been first derived by Poor [6]. Using the Thomae formula and some theta identities, this leads to the following identity for all hyperelliptic Riemann surfaces [6]

$$
F_{g}(\tau)=0,
$$

where $F_{g}(Z)$ is the modular form of weight 8

$$
F_{g}(Z)=2^{g} \sum_{\delta \text { even }} \theta^{16}[\delta](0, Z)-\left(\sum_{\delta \text { even }} \theta^{8}[\delta](0, Z)\right)^{2},
$$

and $Z$ is an arbitrary element of the Siegel upper half-space

$$
\mathfrak{H}_{g}:=\left\{Z \in M_{g}(\mathbb{C}) \mid{ }^{t} Z=Z, \operatorname{Im} Z>0\right\} .
$$

It turns out that $F_{g}$ is proportional to the difference between the theta series associated to the even unimodular lattices $E_{8} \oplus E_{8}$ and $D_{16}^{+}$

$$
F_{g}(Z)=2^{2 g}\left(\Theta_{D_{16}^{+}}(Z)-\Theta_{E_{8}}^{2}(Z)\right) .
$$


Let $\mathcal{I}_{g}$ be the closure of the locus of Riemann period matrices in $\mathfrak{H}_{g} . F_{4}$ is the SchottkyIgusa form $[7,8]$ and the irreducible variety in $\mathfrak{H}_{4}$ defined by $F_{4}=0$ is $\mathcal{I}_{4} \subset \mathfrak{H}_{4}$. This explicitly solves the Schottky problem for $g=4$.

A consequence of the above result is that the effective coupling constants of $N=2$ super Yang-Mills theories with hyperelliptic curves satisfy the relation

$$
\Theta_{D_{16}^{+}}(\tau)=\Theta_{E_{8}}^{2}(\tau)
$$

This means that the effective coupling constants of effective supersymmetric gauge theories do not distinguish between the two lattices defining the two kinds of heterotic string, the heterotic $\mathrm{SO}(32)$ and the heterotic $E_{8} \times E_{8}$ [9]. This suggests a possible new connection between string and SYM theories.

It would be then interesting to understand if (8) extends to all curves describing effective supersymmetric gauge theories. An interesting example is provided by the Gaiotto curves [10]

$$
y^{2}=\frac{\mathcal{P}_{2 n+2}(x)}{x^{2}\left(x-x_{1}\right)^{2} \cdots\left(x-x_{n}\right)^{2}(x-1)^{2}},
$$

where the $x_{i}$ 's denote the punctures, $\mathcal{P}_{2 n+2}(x)$ is a polynomial of degree $(2 n+2)$ depending on the Coulomb branch $u_{i}$, on the masses and on the gauge couplings $q_{i}=x_{i} / x_{i+1}$. Recently, in [11], using the following analogous of the relation in [12]

$$
U_{i}=q_{i} \frac{\partial F}{\partial q_{i}}
$$

the functional dependence of the gauge invariant modulus $U_{i}=\left\langle\operatorname{Tr} \Phi_{i}^{2}\right\rangle$ on the $a_{i}$ 's has been obtained. Integrating (10) with respect to $\log q_{i}$ one obtains the prepotential $F$ [11]. In this way one may compare such an expression with the Nekrasov's prepotential [13]. In turn, all this is related to the recent work $[14,15]$, where it has been proposed to identify the $U_{i}$ 's with the residues of the quadratic differential $y^{2}(x)$ at the punctures.

We now provide some evidence that (8) holds for supersymmetric gauge theories described by a prepotential. We first insist on the crucial role of duality. Let us consider the case of gauge group $\mathrm{SU}(2)$. It is immediately verified that the theory has a basic invariance under the translation of the effective coupling constant. This means that the gauge invariant modulus $u$ has the invariance

$$
u(\tau+2)=u(\tau) .
$$

Denote by $T$ the translation operator by a unit. On the other hand, the theory admits a dual representation. This means that the same theory can be expressed in terms of dual variables, in particular in terms of

$$
\tau_{D}=-\frac{1}{\tau}
$$

Denote by $S$ the inversion operator. Since the dual theory has the same formal properties of the original formulation, it follows that it also remains invariant under $T^{2}$, that is

$$
u\left(\tau_{D}+2\right)=u\left(\tau_{D}\right)
$$


It follows that the trivial $T^{2}$ symmetry, together with the existence of a dual formulation, leads to the fundamental symmetry group generated by

$$
\begin{aligned}
u\left(T^{2} \tau\right) & =u(\tau), \\
u\left(S^{-1} T^{2} S \tau\right) & =u\left(S^{-1} T^{2} \tau_{D}\right)=u\left(S^{-1} \tau_{D}\right)=u(\tau) .
\end{aligned}
$$

Since acting iteratively with $T^{2}$ and $S^{-1} T^{2} S$ we get $\Gamma(2)$, it follows that this is the symmetry group of $N=2 \mathrm{SYM}$ with gauge group $\mathrm{SU}(2)$. This solves the theory and leads to the relation [12]

$$
u=\pi i\left(F-\frac{a}{2} \frac{\partial F}{\partial a}\right) .
$$

A similar construction should lead to a short proof for more general cases. What is important here, is just the existence of a dual formulation of the theory and translation invariance. Existence of the prepotential, the above relation and the existence of a Riemann period matrix, are strictly related facts. This identifies a particular class of Riemann surfaces, namely the ones with period matrix

$$
\tau_{i j}=\frac{\partial^{2} F}{\partial a_{i} \partial a_{j}} .
$$

We note that the presence of punctures in Gaiotto curves may be considered as a suitable limit procedure corresponding to the degeneration of the moduli space of hyperelliptic curves. However, as a direct check one should apply the polynomial identity (4) to the polynomials of Gaiotto's curves, identifies the period matrix $\tau_{i j}$ by (10), and then using the Thomae formulas to verify whether (8) holds in this case. More generally, one should investigate, using the inversion method in [11], if all effective supersymmetric theories admitting the relation (12) may correspond to effective coupling constants satisfying (8).

We conclude observing that a related approach to check (8) is to use the M-theory construction $[16,17]$. We also note that since the underlying theory of Riemann surfaces is the uniformization theory, it follows that Liouville theory should emerge naturally in such an investigation. The role of Liouville theory in supersymmetric Yang-Mills theories has been observed in [12] and [3]. Subsequently, it has been shown that Seiberg-Witten theory can be formulated on the moduli space of punctured spheres, whose geometry is described by the Liouville action [18]. Interestingly, related structures also appear in the AGT correspondence [19, 20].

\section{Acknowledgments}

It is a pleasure to thank Samuel Grushevsky, Daniel Ricci-Pacifici, Giulio Pasini, Paolo Pasti, Riccardo Salvati-Manni, Dmitri Sorokin and Roberto Volpato for interesting discussions.

Open Access. This article is distributed under the terms of the Creative Commons Attribution License (CC-BY 4.0), which permits any use, distribution and reproduction in any medium, provided the original author(s) and source are credited. 


\section{References}

[1] N. Seiberg and E. Witten, Electric-magnetic duality, monopole condensation and confinement in $N=2$ supersymmetric Yang-Mills theory, Nucl. Phys. B 426 (1994) 19 [Erratum ibid. B 430 (1994) 485] [hep-th/9407087] [INSPIRE].

[2] N. Seiberg and E. Witten, Monopoles, duality and chiral symmetry breaking in $N=2$ supersymmetric QCD, Nucl. Phys. B 431 (1994) 484 [hep-th/9408099] [INSPIRE].

[3] G. Bonelli, M. Matone and M. Tonin, Solving $N=2$ SYM by reflection symmetry of quantum vacua, Phys. Rev. D 55 (1997) 6466 [hep-th/9610026] [INSPIRE].

[4] A. Klemm, W. Lerche, S. Yankielowicz and S. Theisen, Simple singularities and $N=2$ supersymmetric Yang-Mills theory, Phys. Lett. B 344 (1995) 169 [hep-th/9411048] [INSPIRE].

[5] P.C. Argyres and A.E. Faraggi, The vacuum structure and spectrum of $N=2$ supersymmetric $\mathrm{SU}(N)$ gauge theory, Phys. Rev. Lett. 74 (1995) 3931 [hep-th/9411057] [INSPIRE].

[6] C. Poor, Schottky's form for and the hyperelliptic locus, Proc. Amer. Math. Soc. 124 (1996) 1987.

[7] J.-I. Igusa, Schottky's invariant and quadratic forms, in E. B. Christoffel. The Influence of His Work on Mathematics and the Physical Sciences, Birkhäuser, Basel (1981), pp. 352-362, http://dx.doi.org/10.1007/978-3-0348-5452-8_24.

[8] J.-I. Igusa, On the irreducibility of Schottky's divisor, J. Fac. Sci. Univ. Tokyo Sect. IA Math. 28 (1981) 531.

[9] D.J. Gross, J.A. Harvey, E.J. Martinec and R. Rohm, The Heterotic String, Phys. Rev. Lett. 54 (1985) 502 [InSPIRE].

[10] D. Gaiotto, N=2 dualities, JHEP 08 (2012) 034 [arXiv:0904.2715] [INSPIRE].

[11] S.K. Ashok, M. Billó, E. Dell'Aquila, M. Frau, R.R. John and A. Lerda, Non-perturbative studies of $N=2$ conformal quiver gauge theories, Fortsch. Phys. 63 (2015) 259 [arXiv: 1502.05581] [INSPIRE].

[12] M. Matone, Instantons and recursion relations in $N=2$ SUSY gauge theory, Phys. Lett. B 357 (1995) 342 [hep-th/9506102] [INSPIRE].

[13] N.A. Nekrasov, Seiberg-Witten prepotential from instanton counting, Adv. Theor. Math. Phys. 7 (2004) 831 [hep-th/0206161] [InSPIRE].

[14] A. Marshakov, Tau-functions for Quiver Gauge Theories, JHEP 07 (2013) 068 [arXiv: 1303.0753] [INSPIRE].

[15] P. Gavrylenko and A. Marshakov, Residue Formulas for Prepotentials, Instanton Expansions and Conformal Blocks, JHEP 05 (2014) 097 [arXiv: 1312.6382] [INSPIRE].

[16] E. Witten, Solutions of four-dimensional field theories via M-theory, Nucl. Phys. B 500 (1997) 3 [hep-th/9703166] [INSPIRE].

[17] J.M. Isidro, On the WDVV equation and M-theory, Nucl. Phys. B 539 (1999) 379 [hep-th/9805051] [INSPIRE]. 
[18] G. Bertoldi, S. Bolognesi, M. Matone, L. Mazzucato and Y. Nakayama, The Liouville geometry of $N=2$ instantons and the moduli of punctured spheres, JHEP 05 (2004) 075 [hep-th/0405117] [INSPIRE].

[19] L.F. Alday, D. Gaiotto and Y. Tachikawa, Liouville Correlation Functions from Four-dimensional Gauge Theories, Lett. Math. Phys. 91 (2010) 167 [arXiv:0906.3219] [INSPIRE].

[20] L.F. Alday, D. Gaiotto, S. Gukov, Y. Tachikawa and H. Verlinde, Loop and surface operators in $N=2$ gauge theory and Liouville modular geometry, JHEP 01 (2010) 113 [arXiv: 0909.0945] [INSPIRE]. 